\title{
Harvest of Product from Whole Cell Lysate
}

National Cancer Institute

\section{Source}

National Cancer Institute. Harvest of Product from Whole Cell Lysate. NCI Thesaurus.

Code C112937.

Collection of a material of interest from a cell lysate. 